\title{
Updates in Gastro-Intestinal Cancer Related Malnutrition
}

\author{
Alaa Ali M. Elzohry MD ${ }^{1 *}$, Khalid Fawzy Mohammed MD², Asmaa Mohamed Aly MSc ${ }^{3}$ \\ ${ }^{1}$ Department of Anesthesia, ICU and pain Relief, South Egypt Cancer Institute, Assiut University \\ ${ }^{2}$ Department of Tropical Medicine and Gastroenterology department, Faculty of Medicine, Assiut University \\ ${ }^{3}$ Department of Anesthesia and ICU, Faculty of Medicine, Minia University, Egypt
}

*Corresponding Author: Alaa Ali M. Elzohry, Department of Anesthesia, ICU and pain Relief, South Egypt Cancer Institute, Assiut University, Email: alaa.zohiry@hotmail.com

\begin{abstract}
Background: Gastro-intestinal cancer is a common malignant tumour affecting mankind and it arise from different part of digestive tract. Hypoalbuminemia and weight loss often result from malnourishment. Malnourished patients have a higher incidence of mortality and morbidity causing prolonged hospital stay.
\end{abstract}

Aim of the Work: To discuss the incidence and effects of Gastro-intestinal cancer related malnutrition and demonstrate updates in management to solve this problem.

Study Design: Narrative review article.

Conclusions The current review revealed that malnutrition related to GIT tumors increases the risk of complications that occurred to patients and increase the duration of stay in hospital and ICU in GIT cancer patients, as such, accounts for burden on ICU budget.

Keywords: Gastro- intestinal cancer Hypoalbuminemia- ICU - major cancer surgery-SOFA score.

\section{BACKGROUND}

The definition of Cancer (this is an aggressive disease that destroys the region of origin and can spread as metastasis or direct extension to other organ, causing multiple complications and progressive loss of organ function). The growth of the cancer affects nutritional status in most cases. [1] Gastro-esophageal cancer is a common malignant tumor of the GIT, and surgical treatment remains the first-line of treatment. [2]

Gastro esophageal cancer is still rare in Western countries, it consider fatal in the vast majority of cases. In the United States, an approximately 17000 new cases were diagnosed in 2015, of them 15000 cases were died from the disease. [3]

The esophagus extends anatomically from the crico-pharyngeal sphincter to the gastro esophageal (GE) junction and divided into three parts; the cervical, upper- to mid-thoracic, and thoracic portions. This is important, because choice of treatment approaches may vary according to the anatomical site of the cancer. It may not be possible to determine the site of origin if the cancer involves the GE junction itself. Despite all advances in treatment modalities and prevention, cancer remains one of the most debilitating diseases nowadays, and considers the second leading cause of death in USA [4].

Malnutrition and weight loss are common complication in GIT cancer patients and may be the first symptom to diagnose the presence of the cancer. And before starting treatment, patients may experience significant metabolic and physiological changes with increased requirement of macro- and micronutrients [5].

\subsection{Prevalence of the Malnutrition}

Prevalence of malnutrition is significantly vary with type of tumor, site, and extension, as well as treatment modalities. And the prevalence ranges between $15 \%$ and $80 \%$. Also the reported in-hospital prevalence of malnourished patients on admission ranges up to 50\%according to some literatures [6]. Malnutrition may affect negatively the surgical decision to resect the tumor or not, which is the main curative line of management of GIT cancer. Malnutrition and weight loss can increase the risk of postoperative complications, such as delayed wound healing, dehiscence of anastomosis line, and mortality [7]. 
The World Health Organization (WHO) classifies malnutrition as a great single risk factor that affects the public health [8]. This led to increasing evidence during recent years of importance of nutritional screening and therapy as adjuncts to modern surgical care because up to $42 \%$ of patients are at risk of malnutrition preoperatively [9].

Medical management of cancer patients means, administration of cytotoxic agents and/or radiation therapy. These modalities can eradicate or decrease tumor size, but usually have several serious side effects that cause weakness the patient, by decreasing appetite or causing nausea and vomiting, fatigue, and asthenia. When malnutrition happened, it is very important to decrease cytotoxic agent's dose and to modify the radiation regimen up to cessation of treatment and this cause reduction in the time of remission and decreasing overall survival rates to radio/chemotherapy ([10-11].

In addition, malnutrition impairs immune system and red decreases the body's defense against infectious microorganisms. So, malnutrition considered a poor prognostic factor and, as such, should be detected early and treated as early as possible [12].

Malnutrition affects the outcome of treatment for cancer (chemotherapy, radiation, and surgery) due to changes in metabolism and pharmacokinetics. Moreover, malnutrition is responsible for changes in drug absorption, protein binding, hepatic metabolism, and renal clearance of drugs metabolites [13]. All these changes may significantly increase the incidence of drug toxicity such as etoposide, cisplatinum, paclitaxel, and irinotecan metabolites [14].

Malnutrition causes depletion of nicotinamide adenine dinucleotide phosphate reserves so decreases the oxidative metabolism of the liver performed by cytochrome P-450 isoenzymes. Other metabolic pathways also impaired, thus prolonging drug half-life. The effect of malnutrition on renal function is less clear, but measurements of inulin clearance in malnourished children showed a reduced glomerular filtration rate (GFR) [15].

\section{Possible Mechanisms of MalnUtrition IN GIT TUMORS}

Malnutrition is more frequent in gastrointestinal (GI), head and neck and lung and it is associated with a poor prognosis. The underlying etiology of malnutrition is multifactorial and includes abnormalities in GI function due to tumorrelated mechanisms or treatment lines in addition to tumor-induced metabolic abnormalities. [16]

\subsection{Tumor-Related Mechanisms}

Many nutritional troubles originate from effects of the tumor locally. Tumors in the gastrointestinal tract cause GIT obstruction, decrease digestion, nausea, vomiting and/or decrease absorption. Also changes in normal metabolism of carbohydrates, protein, and/or fats. [16]

Malnutrition which occurred before surgery is due to decreased oral intake, tumour related cachexia, decreaded absorption due to local effects of CIT tumors. Moreover, low socioeconomic status, as in elderly and handicapped patients, represents an important risk factor. [17-18]

Furthermore, GIT tumors affect organ function, for example, diarrhea in pancreatic and biliary cancer due to lack of digestive enzymes. The presence of visceral pain during eating and digestion is another important factor that causes limitation of oral intake. [19]

Moreover, after surgical removal of GIT tumors, many changes affect the processes of digestion causing, dumping syndrome (in gastric surgery), early satiety or diarrhea (in pancreatic and colonic resection). Each condition of these requires specific dietetic regimens for ideal nutritional rehabilitation. [20]

\subsection{Chemotherapy and Radiation Therapy Related Mechanisms}

Treatment options of GIT cancer (as chemotherapy and radiotherapy) can cause severe GIT symptoms as; anorexia, early satiety, nausea, vomiting, oral and intestinal fungal mucositis that cause dysphagia, diarrhea, hemorroids, anal fissures. All these symptoms may affect food choices and contribute to inadequate meal intake and reduced quality of life. In some cases, co-administered medications, taken to control symptoms or to treat adverse effects associated with anticancer treatments, can produce their own adverse events. [21].

\subsection{Surgery Related Malnutrion}

Di-gestive tract surgery (as laryngectomy, pharyngolaryngectomy, esophagectomy) can interfere with nutrition by effects on swallowing and gastrointestinal motility, or more often by 
malabsorption [22]. The most common complications after oral cancer surgery are alterations in swallowing and mastication that may continue for up to a year after treatment in up to $50 \%$ of the patients. Three years after surgery, $42 \%$ of laryngectomy and $50 \%$ of pharyngolaryngectomy patients experienced long-term dysphagia leading to nutritional deficits and requiring a modification of their diet or tube feeding. Chronic xerostomia, that also impairs swallowing, can occur in patients up to 5-years after treatment. [23-24]

Dysphagia, heartburn, hoaresness, reflux, abnormal gastric emptying, dumping syndrome (diarrhoea, abdominal cramps, nausea, dizziness, diaphoresis), increased stool frequency are frequently reported after esophagectomy; $80-90 \%$ of the patients experience early satiety, about $75 \%$ suffer from post-prandial dumping syndrome, $50-60 \%$ have reflux of food/fluid and absence of hunger in the first postoperative year. Weight loss is common after esophagectomy, and the majority of patients are unable to return to their preoperative weight. [25]

Gastrectomy. Total gastrectomy has a profound effect on nutritional status, including weight loss, malabsorption, mal-digestion, shortened intestinal transit time and bacterial over-growth. This surgical intervention, by reducing the reservoir into which patients can eat and by altering the physiology of digestion produces malnutrition in approximately $80 \%$ of the patients [3]. Diarrhoea usually occurs within 1$2 \mathrm{~h}$ after eating, and is probably partly caused by vagotomy, lack of gastric hormones, and defective fat absorption due to pancreatic insufficiency. [26]

Factors increasing malabsorption include defective stimulation of biliary and pancreatic secretions by ingested food by-passing the duodenum, and inadequate mixing of biliary and pancreatic secretions with food. Malabsorption of dietary fat has been proposed as a major contributor to weight loss. Malabsorption of aminoacids has also been reported, resulting in a state of persistent proteolysis for long periods after surgery [3]. Loss of gastric and duodenal absorptive surface causes malabsorption of iron, vitamin B12, calcium, fat and carotene; stasis in the afferent loop can lead to bacterial overgrowth and abnormalities in bile salt metabolism [27].

Pancreaticoduodenectomy. The degree of pancreatic func-tion impairment is related to the extent of parenchyma resection and the functional state of the residual pancreas. Accordingly with what was just previously mentioned, another contributing factor for malnutrition is disease progression. One year after curative resection, the malnutrition status is main-tained as well as many gastrointestinal symptoms. [28-29]

Therapy is based on the oral administration of pancreatic enzymes aiming at providing the duodenal lumen with sufficient active lipase at the time of gastric emptying of nutrients. Foods containing long chain triglycerides (LCT) may be replaced by the recruitment of medium chain triglycerides (MCT) that are more rapidly hydrolyzed and absorbed. MCT should be given in four daily doses of about $15 \mathrm{~mL}$, while higher doses are not recommended for the possible induction of diarrhea; there can be loss of endocrine function too, and when glucose is not well controlled, there will be weight loss. [30]

\subsection{Treatments of Malnutrition in Patients with GIT Cancer}

Nutrition plays major roles in many aspects of cancer development and treatment [31]. Malnutrition is a common problem in cancer patients that has been recognized as an important component of adverse outcomes, including increased morbidity and mortality and decreased quality of life. Weight loss has been identified as an indicator of poor prognosis in cancer patients. It has been shown that at the time of diagnosis, $80 \%$ of patients with upper gastrointestinal cancer and $60 \%$ of patients with lung cancer have already experienced a significant weight loss, [32]

Generally defined as at least a $10 \%$ loss of body weight in 6 months' time [33]. Good nutrition practices can help cancer patients maintain weight and the body's nutrition stores, offering relief from nutrition impact symptoms and improving quality of life. Poor nutrition practices, which can lead to under nutrition, can contribute to the incidence and severity of treatment side effects and increase the risk of infection, thereby reducing chances for survival [34].

Nutrition impact symptoms are those symptoms that impede oral intake. They include, but are not limited to, anorexia, nausea, vomiting, diarrhea, constipation, stomatitis, mucositis, dysphagia, alterations in taste and smell, pain, depression, and anxiety [35]. 
Early recognition and detection of risk for malnutrition through nutrition screening followed by comprehensive assessments is increasingly recognized as imperative in the development of standards of quality of care in oncology practices. Undesirable weight gain may be an effect of chemotherapy treatment for early-stage cancers, possibly resulting from decreases in resting metabolism. Consequently; the eating practices of individuals diagnosed with cancer should be assessed throughout the continuum of care to reflect the changing goals of nutritional therapy [36]

\subsection{Nutritional Options in Patients with GIT Cancer}

Nutrients are macromolecules (fat, proteins, lipid and water), micro molecules (vitamins, minerals, electrolytes and trace elements) and more important components of immunemodulating artificial nutrition as:

- Glutamine

- Arginine

- N-acetyl cysteine (as a cysteine precursor)

- Branched chain amino acids

- Nucleotides

- Long-chain n-3 fatty acids

- Antioxidant vitamins

- Trace elements

- Taurine

Immunomodulatory substance interfere with 3 basic areas of the immune responses directly or indirectly;

(i) The mucosal barrier function

(ii) The cellular defence function and

(iii) The local or systemic inflammatory response. [37]

Nutrition support is an important therapeutic intervention for improving outcomes in hospitalized patients. Nutrition support has evolved substantially over the past 20 years, from a primarily supportive strategy to an active therapeutic intervention. Understanding of nutrient effects upon disease processes has led to the development of specialized nutritional formulas. One class of these formulas are called immune modulating diets, or immune modulating diets (IMDs). [38]

\section{DISCUSSION}

An impressive number of studies have demonstrated that perioperative arginine/omega-
3 immunonutrition in patients with esophageal, gastric, colonic and head and neck cancer patients improves short-term outcomes, such as local wound complications, fistula rates, and length of hospital stay [39]

Immunonutrition contain pharmacologic doses of nutrients including arginine (Arg), $\omega-3$ polyunsaturated fatty acids ( $\omega-3$ PUFA), glutamine (Glu) and ribonucleic acid (RNA). All are proved to enhance immune function in vitro and animal experiments. Some clinical trials has been reported to affect the risk of postoperative infection and length of hospital stay in patients underwent operation. But the outcome of these studies is inconsistent and new sufficient clinical evidences is absent for gastrointestinal surgery. [40]

Glutathione plays a pivotal role as it acts directly as an antioxidant and maintains other components of defence in a reduced state. It has more specific effect on the function of lymphocytes via the thioredoxin system. [41]

Parenteral nutrition has been in use since the 1960s. Total parenteral nutrition (TPN) involves providing all of patient's nutritional needs intravenously and is lifesaving in clinical settings in which adequate enteral based delivery of such nutrition is not possible. It has been one of the most promising modalities of nutrition in neonates, older pediatric patients, and adults with lost or impaired gut function. TPN infusion therapy has grown enormously over the last few decades. There are tens of thousands of patients worldwide permanently dependent on parenteral nutrition (PN) for survival. TPN, a major therapeutic advance in the modern medical era, stil provides ample opportunity for continued exploration. [42-44]

In addition to a more recent study conducted by Thieme et al., 2013 to investigate methods of evaluation of the nutritional status that better correlate with postoperative complications and the length of hospital stay in patients submitted to gastrointestinal or abdominal wall surgeries. They reported that the immunologic status, analysed by total lymphocytes count was not correlated to postoperative outcomes, suggesting that this is not an adequate parameter to predict postoperative complications. [45]

Chandrasinghe et al. 2013, showed a significant association between a low preoperative serum albumin of less than $35 \mathrm{~g} / \mathrm{L}$, with a reduction in overall survival for patients undergoing surgery for rectal cancer. They concluded that albumin 
can be used as a cost effective and a sensitive marker to predict survival in rectal cancer compared to other available inflammatory markers. [46]

According to Campos et al., 2008, several factors affect post-surgical wound healing such as protein malnutrition that can adversely affect wound healing. Collagen is the most abundant protein in human body and provides strength, integrity and structure. Besides protein, wound healing is stimulated by an adequate amount of energy, vitamins A, C, E and B complex, zinc, cooper, and selenium. Malnutrition influence in wound healing quality and duration and serum proteins, such as albumin, are important to this process. [47]

\section{CONClusion}

In conclusion, the current review revealed that malnutrition increases the risk of complications and length of stay in hospital in GIT cancer patients, as such, accounts for high proportion of ICU budget.

We believe that the current review give reasons for raising attention about using serum albumin level nutritional status as a simple and low-cost prognostic tool to predict outcome and consequently, decreasing the incidence of complications GIT cancer patients.

\section{REFERENCES}

[1] Pedrosa IV, Burgos M, Souza NC, et al. Aspectos nutricionais em obesos antes e após a cirurgia bariátrica. Rev Col Bras Cir. 2009; 36(4):316-22.

[2] M. I. T. D. Correia and D. L. Waitzberg, "The impact of malnutrition on morbidity, mortality, length of hospital stay and costs evaluated through a multivariate model analysis," Clinical Nutrition, vol. 22, no. 3, pp. 235-239, 2003.

[3] K. Norman, C. Pichard, H. Lochs, and M. Pirlich, "Prognostic impact of disease-related malnutrition," Clinical Nutrition, vol. 27, no. 1, pp. 5-15, 2008.

[4] D. L. Waitzberg, W. T. Caiaffa, and M. I. T. D. Correia, "Hospital malnutrition: the Brazilian national survey (IBRANUTRI): a study of 4000 patients," Nutrition, vol. 17 , no. 7-8, pp. 573580, 2001.

[5] F. Bozzetti, L. Gianotti, M. Braga, V. Di Carlo, and L. Mariani, "Postoperative complications in gastrointestinal cancer patients: the joint role of the nutritional status and the nutritional support," Clinical Nutrition, vol. 26, no. 6, pp. 698-709, 2007.

[6] J. Sorensen, J. Kondrup, J. Prokopowicz et al., "EuroOOPS: an international, multicentre study to implement nutritional risk screening and evaluate clinical outcome," Clinical Nutrition, vol. 27, no. 3, pp. 340-349, 2011.

[7] M. Correia, M. Cravo, P. Marques-Vidal et al., "Serum concentrations of TNF-alpha as a surrogate marker for malnutrition and worse quality of life in patients with gastric cancer," Clinical Nutrition, vol. 26, no. 6, pp. 728-735, 2009.

[8] F. Bozzetti, M. Braga, L. Gianotti, C. Gavazzi, and L. Mariani, "Postoperative enteral versus parenteral nutrition in malnourished patients with gastrointestinal cancer: a randomised multicentre trial," Lancet, vol. 358, no. 9292, pp. 1487-1492, 2011.

[9] Y. Zheng, F. Li, B. Qi et al., "Application of perioperative immunonutrition for gastrointestinal surgery: a meta-analysis of randomized controlled trials," Asia Pacific Journal of Clinical Nutrition, vol. 16, supplement 1, pp. 253-257, 2007.

[10] Reeves GK, Pirie K, Beral V, et al.: Cancer incidence and mortality in relation to body mass index in the Million Women Study: cohort study. BMJ 335 (7630): 1134, 2007.

[11] Wojtaszek CA, Kochis LM, Cunningham RS: Nutrition impact symptoms in the oncology patient. Oncology Issues 17 (2): 15-7, 2012.

[12] Langstein HN, Norton JA: Mechanisms of cancer cachexia. Hematol Oncol Clin North Am 5 (1): 103-23, 1991.

[13] Ottery FD: Cancer cachexia: prevention, early diagnosis, and management. Cancer Pract 2 (2): 123-31, 1994 Mar-Apr.

[14] Tisdale MJ: Biology of cachexia. J Natl Cancer Inst 89 (23): 1763-73, 1997.

[15] Strasser F, Bruera ED: Update on anorexia and cachexia. Hematol Oncol Clin North Am 16 (3): 589-617, 2002.

[16] Gambardella A, Tortoriello R, Tagliamonte MR, et al.: Metabolic changes in elderly cancer patients after glucose ingestion. The role of tumor necrosis factor-alpha. Cancer 79 (1): 177-84, 2007.

[17] Ottery FD: Cancer cachexia: prevention, early diagnosis, and management. Cancer Pract 2 (2): 123-31, 2009 Mar-Apr.

[18] Kutynec CL, McCargar L, Barr SI, et al.: Energy balance in women with breast cancer during adjuvant treatment. J Am Diet Assoc 99 (10): 1222-7, 2014.

[19] Bosaeus I, Daneryd P, Svanberg E, et al.: Dietary intake and resting energy expenditure in relation to weight loss in unselected cancer patients. Int J Cancer 93 (3): 380-3, 2011. 
[20] Murphy S, Von Roenn JH: Pharmacological management of anorexia and cachexia. In: McCallum PD, Polisena CG, eds.: The Clinical Guide to Oncology Nutrition. Chicago, Ill: The American Dietetic Association, 2015, pp 12733.

[21] Kanda M, Fujii T, Kodera Y, et al. Nutritional predictors of postoperative outcome in pancreatic cancer. British Journal of Surgery 2011; 98:268-274.

[22] Jones AE, Trzeciak S, Kline JA. The Sequential Organ Failure Assessment score for predicting outcome in patients with severe sepsis and evidence of hypoperfusion at the time of emergency department presentation. Crit Care Med 2009; 37(5):1649-54.

[23] Wiedermann CJ, Wiedermann W, Joannidis M. Hypoalbuminemia and acute kidney injury: a meta-analysis of observational clinical studies. Intensive Care Med. 2010; 36:1657 -1665

[24] Shitanshu U, Ahmed AN, Laurel W. Rice, et al. Preoperative hypoalbuminemia is an independent predictor of poor perioperative outcomes in women undergoing open surgery for gynecologic malignancies. Gynecologic Oncology 131 (2013) 416-422.

[25] Mizock BA. Immunonutrition and critical illness: an update. Nutrition 2010; 26(7-8):7017.

[26] Braga M. The Award for General Nutrition. Immunonutrition: from laboratory to clinical practice. Nutrition 2007; 23(4):368-70.

[27] Mongardon N, Singer M. The evolutionary role of nutrition and metabolic support in critical illness. Crit Care Clin 2010; 26(3):443-50.

[28] Ariel A, Serhan CN. Resolvins and protectins in the termination program of acute inflammation. Trends Immunol 2007; 28(4):176-83.

[29] Dewar D, Moore FA, Moore EE, et al. Postinjury multiple organ failure. Injury 2009; 40(9):912-8.

[30] Marik PE. Critical illness related corticoseroid insufficiency. Chest 2009; 135(1):181-93.

[31] Elenkov IJ, Kvetnansky R, Hashiramoto A, et al. Low- versus high-baseline epinephrine output shapes opposite innate cytokine profiles: presence of Lewis- and Fischer-like neurohormonal immune phenotypes in humans? J Immunol 2008; 181(3):1737-45.

[32] 31-Zhu X, Herrera G, Ochoa JB. Immunosupression and infection after major surgery: A nutritional deficiency. Crit Care Clin 2010; 26(3):491-500.

[33] Braitch M, Harikrishnan S, Robins RA, et al. Glucocorticoids increase CD4CD25 cell percentage and Foxp3 expression in patients with multiple sclerosis. Acta Neurol Scand 2009; 119(4):239-45.
[34] Mizota T, Fujita-Kambara C, Matsuya N, et al. Effect of dietary fatty acid composition on Th1/Th2 polarization in lymphocytes. JPEN J Parenter Enteral Nutr 2009; 33(4):390-6.

[35] Suzuki D, Furukawa K, Kimura F, et al. Effects of perioperative immunonutrition on cellmediated immunity, T helper type 1 (Th1)/Th2 differentiation, and Th17 response after pancreaticoduodenectomy. Surgery 2010; 148(3):573-81.

[36] Klek S, Sierzega M, Szybinski P, et al. The immunomodulating enteral nutrition in malnourished surgical patients - a prospective, randomized, double-blind clinical trial. Clin Nutr 2011; 30(3):282-8.

[37] Mudge L, Isenring E, Jamieson GG. Immunonutrition in patients undergoing esophageal cancer resection. Dis Esophagus 2011; 24(3):160-5.

[38] Buijs N, van Bokhorst-de van der Schueren MA, Langius JA, et al. Perioperative argininesupplemented nutrition in malnourished patients with head and neck cancer improves long-term survival. Am J Clin Nutr 2010; 92(5):1151-6.

[39] Norian LA, Rodriguez PC, O’Mara LA, et al. Tumor-infiltrating regulatory dendritic cells inhibit CD8+ $\mathrm{T}$ cell function via $\mathrm{L}$-arginine metabolism. Cancer Res 2009; 69(7):3086-94.

[40] Rodriguez PC, Ochoa AC. Arginine regulation by myeloid derived suppressor cells and tolerance in cancer: mechanisms and therapeutic perspectives. Immunol Rev 2008; 222:180-91.

[41] Cockbain AJ, Toogood GJ, Hull MA. Omega-3 polyunsaturated fatty acids for the treatment and prevention of colorectal cancer. Gut 2012; 61(1):135-49.

[42] Murphy RA, Mourtzakis M, Chu QS, et al. Nutritional intervention with fish oil provides a benefit over standard of care for weight and skeletal muscle mass in patients with nonsmall cell lung cancer receiving chemotherapy. Cancer 2011; 117(8):1775-82.

[43] Puja Dullo, Neeraj Vedi. Importance of immunonutrients. Pak J Physiol 2010;6(1)

[44] Calder PC. Immunonutrition. Brit Med J 2003; $327,117-118$.

[45] Rodriguez PC, Ernstoff MS, Hernandez C, et al. Arginase I-producing myeloid-derived suppressor cells in renal cell carcinoma are a subpopulation of activated granulocytes. Cancer Res 2009; 69(4):1553-60.

[46] Thieme RD, Cutchma G, Chieferdecker ME, et al. Nutritional risk index is predictor of postoperative complications in operations of digestive system or abdominal wall? Arq Bras Cir Dig 2013;26 (4):286-292. 
[47] Chandrasinghe C, Dileepa S, Sumudu K, et al. Pre-operative hypoalbuminaemia predicts poor overall survival in rectal cancer: a retrospective cohort analysis. BMC Clinical Pathology 2013, 13:12.
[48] Campos AC, Groth AK, Branco AB. Assessment and nutritional aspects of wound healing. Curr Opin Clin Nutr Metab Care. 2008; 11:281-8.

Citation: Alaa Ali M. Elzohry, Khalid Fawzy Mohammed, Asmaa Mohamed Aly. Updates in GastroIntestinal Cancer Related Malnutrition. ARC Journal of Anesthesiology. 2019; 4(1):10-16. DOI: dx.doi.org/ 10.20431/2455-9792.0401002.

Copyright: (C) 2019 Authors. This is an open-access article distributed under the terms of the Creative Commons Attribution License, which permits unrestricted use, distribution, and reproduction in any medium, provided the original author and source are credited. 